\title{
OPEN REDUCTION AND INTERNAL FIXATION OF POSTERIOR PILON FRACTURES WITH BUTTRESS PLATE
}

\author{
Da-wel Chen $^{1}$, Bing LI ${ }^{1}$, Ashwin Aubeeluck ${ }^{1}$, Yun-feng Yang $^{1}$, JiA-QIan Zhou ${ }^{1}$, Guang-rong Yu ${ }^{1}$
}

\section{ABSTRACT}

Objective: Posterior pilon fractures are rare injuries and have not yet gained well recognition. The purpose of this study was to present the treatment outcome for patients with posterior pilon fractures treated with buttress plate. Method: In this retrospective study we identified patients with posterior pilon fractures of the distal tibia who had undergone open reduction and internal fixation at our institute. Between January 2007 and December 2009, 10 patients (mean age, 46.5 years) who had undergone buttress plating via either a posterolateral approach or a dual posterolateral-posteromedial approach, were selected. All 10 patients were available for follow-up. The clinical outcome was evaluated with the American Orthopaedic Foot and Ankle Society (AOFAS) ankle-hindfoot score and the visual analogue sca- le (VAS). The radiological evaluation was performed using the osteoarthritis-score (OA-score). Results: Satisfactory reduction and stable fixation were accomplished in all patients. At a mean follow-up of 36.2 months, all patients had good radiological results and showed satisfactory clinical recovery. The mean AOFAS sore was 87.8, the mean OA-score was 0.6, and the mean VAS scores during rest, active motion, and weight-bearing walking were $0.6,0.8$, and 1.4 , respectively. Conclusion: Buttress plating for posterior pilon fractures gave satisfactory clinical outcomes. It also ensured rigid fixation which in turn enabled earlier postoperative mobilization. Level of Evidence IV, Retrospective Study.

Keywords: Tibial fractures. Ankle injuries. Internal fixators. Bone plates.

Citation: Chen DW, Li B, Aubeeluck A, Yang YF, Zhou JQ, Yu GR. Open reduction and internal fixation of posterior pilon fractures with buttress plate. Acta Ortop Bras. [online] 2014;22(1):48-53. Available from URL: http://www.scielo.br/aob.

\section{INTRODUCTION}

Intra-articular fractures with involvement of the posterior tibial plafond usually bear a worse long-term prognosis and pertain a higher risk of degenerative changes. ${ }^{1,2}$ The mechanism of injury has two components: a vertical compressive component and a torsional component, both individually having the potential to displace a posterior malleolar fragment. Torsional forces will usually displace a small posterior malleolar fragment without involving the articular cartilage, whereas compressive forces can not only displace larger posterior malleolar fragments but also cause proximal impaction of that fragment with formation of step off within the tibial plafond. ${ }^{3,4}$ It seems only reasonable to categorize fractures with large impacted posterior malleolar fragments as pilon fractures.

The notion of posterior pilon fractures has not yet gained recognition. In 1996, Huber et al. ${ }^{5}$ used "trimalleolar pilon fractures" to describe displaced posterior malleolar fractures with cranial migration of the talus and articular impaction of the distal tibia. They thought it was inappropriate to classify these fractures as malleolar fractures and emphasized on the mechanism of axial loading. In 2000, Hansen ${ }^{6}$ introduced the term "posterior pilon" to describe severe trimalleolar fractures with the presence of a fourth fragment located deeper than the avulsed posterior fragment. In agreement with Hansen ${ }^{6}$, Amorosa et al. ${ }^{3}$ thought a posterior pilon fracture resulted from combined forces was a pilon variant in between a low-energy rotational malleolar fracture and a high-energy axially loaded pilon fracture.

Incidence of posterior pilon fractures still remains unclear. In the literature, posterior pilon fractures were often grouped with trimalleolar fractures. ${ }^{5,7-10}$ In a series of 126 consecutive pilon fractures, Topliss et al. ${ }^{11}$ described anatomical features of the fragments of the distal tibia and found that the posterior split-type fractures, i.e. posterior pilon fractures, accounted for $5.6 \%$ (6/108) of all 108 pilon fractures assessed by CT scans.

With regard to the treatment, there is still no consensus as to the optimal solution for posterior pilon fractures in the literature. As one unique type of pilon fractures, the posterior pilon fractures require anatomical reduction and stable fixation. However, different operative techniques including indirect anteroposterior screw-fixation, direct posteroanterior screw-fixation, and but-

All the authors declare that there is no potential conflict of interest referring to this article.

Department of Orthopaedics, Tongji Hospital, Tongji University School of Medicine, Shanghai, China

Work conducted in Department of Orthopaedics, Tongji Hospital, Tongji University School of Medicine, Shanghai, China.

Mailing address:Guang-rong Yu. Department of Orthopaedics, Tongji Hospital, Tongji University School of Medicine, 389 Xincun Road, Shanghai 200065, China. yuguangrong2012@gmail.com 
tress plate fixation have been adopted by different orthopaedic surgeons. ${ }^{3,5}$ In this article, through a retrospective study, we present the treatment outcomes of 10 patients with posterior pilon fractures treated with buttress plate.

\section{PATIENTS AND METHODS}

During a 3-year period from January 1, 2007, to December 31, 2009, 157 consecutive patients with ankle fractures underwent operative treatment at our institution. Of these fractures, 10 fractures in 10 patients with impaction of the posterior tibial plafond were identified as posterior pilon fractures by CT scans and were treated with buttress plating. Those fractures with no impaction of the plafond or treated only by screws were excluded from this study cohort. There were seven males and three females with an average age of 46.5 (range, 21 to 71) years. Four patients had been injured in motor vehicle accidents, three patients had fallen from less than 2-meter height, two patients had had twisting injuries upon falling from more than one step height while going down stairs, and one patient had a history of slip and fall at ground level only. Radiographs of the ankle joint with anteroposterior, mortise and lateral views were taken to evaluate the fractures. Three-dimensional reconstruction of CT scan images was also used to identify the fracture patterns. The transverse CT scan images revealed that the fracture lines extended from the posterior malleolus to the medial malleolus in all cases. Six of the 10 patients had associated complete medial malleolar fractures involving both the anterior and the posterior colliculi. Associated lateral malleolar fractures also occurred in all cases. In the emergency department, all fractures received closed reduction and fixation with plaster splints. Calcaneal traction was applied in three cases and external fixator in two cases because each of these fractures had a concomitant closed Tscherne grade 2 soft-tissue injury. ${ }^{12}$ Definitive fixation was delayed until the soft tissue swelling had subsided. The mean time from injury to operation was 7.8 (range, 6 to 10) days. Patient's data are presented in Table 1.

Ethical approval was obtained from the Human Research Ethics Committee, Tongji Hospital, Tongji University School of Medicine, Shanghai, China. The patients had signed an informed consent form authorizing the use of their clinical data in this study.

\section{Surgical Techniques}

The surgeries were performed under either general or epidural anesthesia. Patients were positioned prone with a bolster under the distal lower leg as prevision for reduction of the talus. A tourniquet was routinely applied on the thigh. A standard posterolateral approach was used. The incision was made midway between the fibula and the lateral border of the Achilles tendon. Dissection in the subcutaneous plane was performed with great care to identify and protect the sural nerve. Deep dissection proceeded through the interval between the peroneal tendons and the flexor hallucis longus tendon. The fibular fracture was exposed by retracting the peroneal tendons laterally and the posterior surface of the distal tibia was reached by retracting the flexor hallucis longus tendon and the deep posterior compartment medially. We preferred to reduce and fix the fibular fracture first. Prior to fixation, the fracture fragments of the tibia and fibula were mobilized using a periosteal elevator so as to ease reduction and the fracture hematoma was removed. The posterior malleolar fragment was then elevated and flipped over to expose the central impacted fragment. Care was taken to preserve the posterior inferior tibiofibular ligament attached to this fragment. After fixation of the fibular fracture, the central impacted fragment was reduced by using a small periosteal elevator or a curette as a joystick. The bone defect was filled with bone allograft. A $1.5 \mathrm{~mm}$ Kirschner wire was used to drill through the central fragment, the anterior cortex of the distal tibia, and the anterior soft-tissue on the opposite side until the tail end of the K-wire was in a position just adequate for temporary fixation of the impacted fragment without affecting reduction of the posterior malleolar fragment. (Figure 1) Then the posterior malleolar fragment was reduced and fixed temporarily with 2-mm K-wires. Large impacted osteochondral fragments were anatomically reduced and inserted press-fit together with the posterior fragment. The nonviable small comminuted ones were removed. It was important to have a detailed appreciation of the fracture morphology and to visualize the proximal part of the posterior malleolar fragment, which served as the principal anatomical reference for anatomical reduction. Once the posterior fragment of the tibia had been reduced, the joint surface could not be visualized directly anymore. After satisfactory fracture reduction was achieved and confirmed with intraoperative fluoroscopy, an appropriately sized buttress plate (1/3 tubular plate, small profile T-type plate, etc.) was screwed to the posterior surface of the distal tibia to fixate the posterior malleolus and prevent its secondary migration. Depending on the degree of comminution of the fragment, an additional screw would sometimes be used to achieve better outcome. (Figure 2)

Table 1. Patient Data.

\begin{tabular}{|c|c|c|c|c|c|c|c|}
\hline Patient No. & Age (yr), Gender & Mechanism & $\begin{array}{l}\text { Associated } \\
\text { Lesion }\end{array}$ & $\begin{array}{c}\text { Syndesmotic screw } \\
\text { fixation }\end{array}$ & Fixation of PTPF & Approach & $\begin{array}{c}\text { Time from Injury to } \\
\text { Surgery (d) }\end{array}$ \\
\hline 1 & $53, M$ & MVA & LMF, WMMF & No & Plate, Screw & PL, PM & 7 \\
\hline 2 & $47, \mathrm{M}$ & FOS & LMF, WMMF & No & Plate & PL, PM & 6 \\
\hline 3 & $21, M$ & FFH & LMF & No & Plate, Screw & $\mathrm{PL}$ & 7 \\
\hline 4 & $34, \mathrm{~F}$ & MVA & LMF, WMMF & No & Plate & PL, PM & 8 \\
\hline 5 & $63, \mathrm{M}$ & FOS & LMF & Yes & Plate & $\mathrm{PL}$ & 9 \\
\hline 6 & $36, \mathrm{M}$ & $\mathrm{FFH}$ & LMF, WMMF & No & Plate & $\mathrm{PL}, \mathrm{PM}$ & 7 \\
\hline 7 & $52, M$ & MVA & LMF & No & Plate & $\mathrm{PL}$ & 10 \\
\hline 8 & $71, \mathrm{~F}$ & GLF & LMF, WMMF & No & Plate, Screw & $\mathrm{PL}, \mathrm{PM}$ & 6 \\
\hline 9 & $46, M$ & $\mathrm{FFH}$ & LMF & No & Plate & $\mathrm{PL}$ & 8 \\
\hline 10 & $42, F$ & MVA & LMF, WMMF & No & Plate & PL, PM & 10 \\
\hline
\end{tabular}



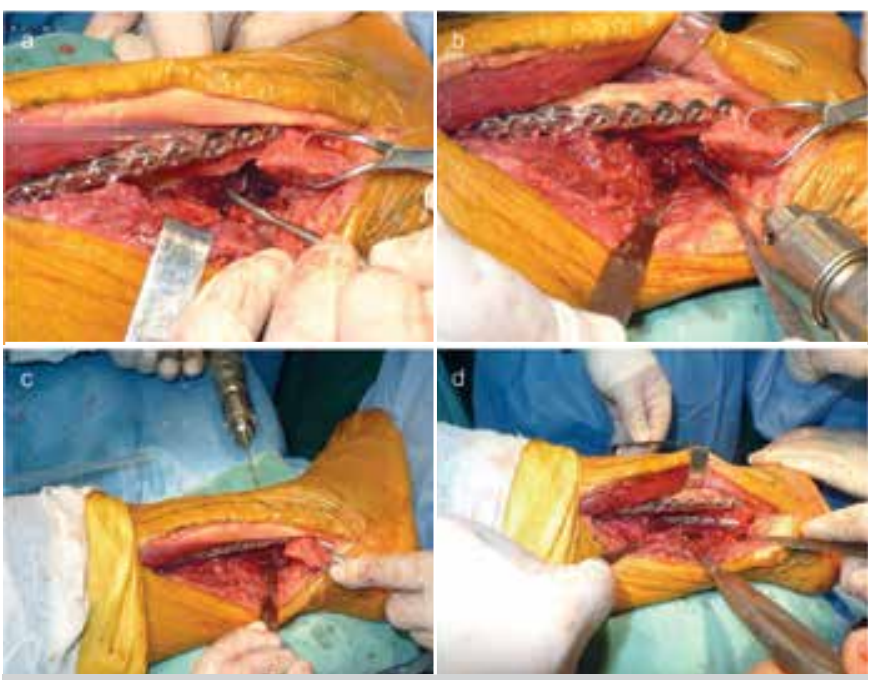

Figure 1. Reduction and fixation of the impacted osteochondral fragment. (a) The central impacted fragment was reached by elevating the posterior fragment and reduced by a small periosteal elevator. (b) A $1.5 \mathrm{~mm} \mathrm{~K}$-wire was used to fix the impacted fragment temporarily. (c) The K-wire was pulled out from the anterior aspect. (d) An 1/3 tubular plate was used to fix the posterior fragment.
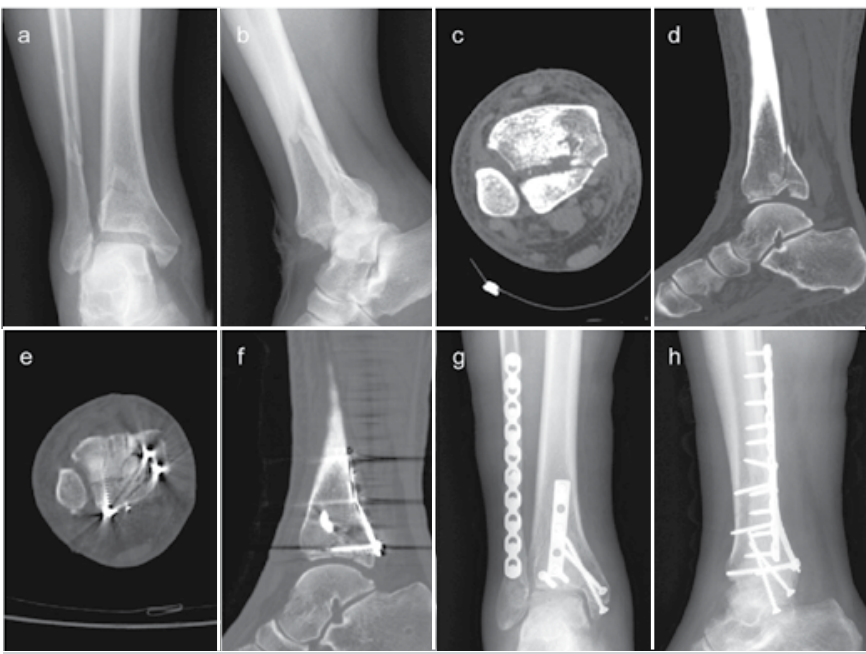

Figure 2. A 53-year-old man was injured in a motor vehicle accident (case 1). (a-b) Fractures of the posterior malleolus can be identified by the anteroposterior and lateral radiographs. (c) Axial CT scan shows comminution of the posterior plafond. (d) Impacted fragment and proximal displacement of the posterior fragment can be identified on the sagittal CT scan. (e) Postoperative sagittal CT scan shows anatomical reduction of the posterior plafond. (f) Postoperative axial CT scan shows anatomical reduction of the syndesmosis. ( $g$-h) Postoperative anteroposterior and lateral radiographs show fracture reduction and internal fixation.

In the presence of a posteromedial fragment needing fixation, an additional posteromedial approach was required. The posteromedial incision was made along the course of the posterior tibial tendon and followed the posteromedial border of the distal tibia and medial malleolus. The flexor retinaculum was incised. Deep dissection continued through the interval between the posterior tibial tendon and the tendon of the flexor digitorum longus. The posterior tibial tendon was retracted anteriorly over the medial malleolus. The tendon of the flexor digitorum longus was retracted posteriorly to protect the neurovascular bundle. An arthrotomy was made to expose the posteromedial fracture fragment. After anatomical reduction was confirmed, depending on the size of the fragment, a small buttress plate or lag screw was used to fix the posteromedial fragment. (Figure 3) Sometimes, the posteromedial fragment was fixed together with the posterolateral fragment using the same plate. In some cases with complete medial malleolar fractures, through the same posteromedial incision, the tibialis posterior tendon was retracted posteriorly to expose the medial malleolus. Then the medial malleolus was reduced and stabilized with lag screws. Flexion of the knee and internal rotation of the limb proved to be of great help during the operation.

After fracture fixation, syndesmotic stability was checked by the Cotton test, and a syndesmotic screw was routinely used if an unstable syndesmosis was found. When good reduction and acceptable hardware placement had been confirmed by intraoperative imagery, wound closure was performed in the usual manner.

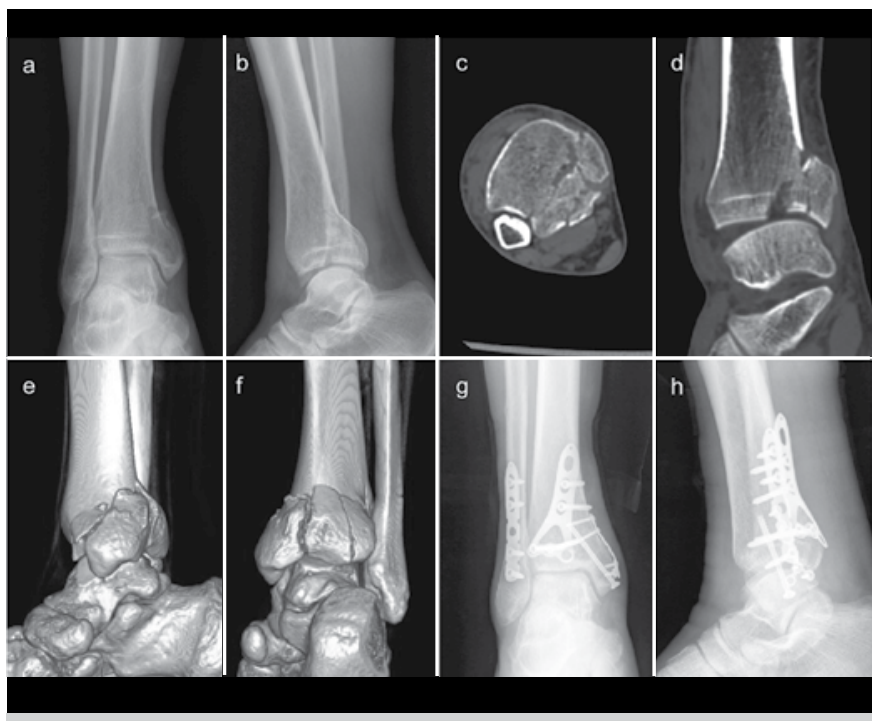

Figure 3. A 36-year-old man was injured by falling from height (case 6). (ab) Preoperative anteroposterior and lateral radiographs. (c) Comminution of the posterior plafond can be identified by the axial CT scan. (d) Proximal displacement of the posterior fragment and subluxation of the talus can be identified on the sagittal CT scan. (e-f) 3-D CT reconstruction showed comminution of the posterior fragments. (g-h) Postoperative anteroposterior and lateral radiographs show fracture reduction and internal fixation.

\section{Postoperative Management}

The postoperative rehabilitation protocol was standardized. No external splints were used. Active range of motion exercises, with the extent gradually increased, were begun after 24 hours. The sutures were removed after 2 weeks. Partial weight bearing was begun at 6 weeks. Full weight bearing was allowed at 3 months when advanced signs of union were seen on radiographs.

\section{Postoperative Assessment}

The quality of fracture reduction was assessed with the immediate postoperative CT scans. Less than $1 \mathrm{~mm}$ articular stepoff was considered as an anatomical reduction. During the period of follow-up, radiographs were taken monthly in the first 3 postoperative months. Thereafter, patients were generally followed 
every 3 months, which changed to every 6 months one year later. At the $24^{\text {th }}$ month of follow-up, the functional outcome was evaluated with the American Orthopaedic Foot and Ankle Society (AOFAS) ankle-hindfoot score., 11,14 The visual analogue scale (VAS) (0, pain-free; 10 , the most unbearable pain) was used to evaluate pain at fracture site during rest, active movement, and weight-bearing walking. ${ }^{2,14-16}$ The radiological evaluation was performed using osteoarthritis-score (OA-score), ${ }^{2,10,14} \mathrm{~A}$ score of 0 was a normal joint; a score of 1 was assigned to the presence of osteophytes without joint space narrowing; a score of 2 was assigned to joint space narrowing with or without osteophytes; and a score of 3 was assigned with sub or total disappearance or deformation of the joint space.

\section{RESULTS}

Impaction of the posterior tibial plafond was found in all cases. The posterolateral approach was used in all patients. A combined approach (posteromedial and posterolateral) was used in six patients. A syndesmotic screw was used in one case. Immediate postoperative CT scanning was performed for all patients. Anatomical reduction was achieved in eight patients. Two patients had a $1 \mathrm{~mm}$ stepoff of the articular surface. All ten patients were available for follow-up at an average of 36.2 (range, 24 to 52) months. The wounds of all patients healed uneventfully. All fractures healed within 13 weeks, without loss of reduction and hardware failure. No infections and nerve injuries occurred. The buttress plate did not cause discomfort in any of our patients. At two years postoperatively, the mean OA-score was 0.6 (range, 0 to 2) in all patients. Four patients had an OA-score of 1 and one patient had an OA-score of 2. The mean AOFAS score was 87.8 (range, 82 to 98). The mean VAS score for fracture pain under different conditions was 0.6 (range, 0 to 2) during rest, 0.8 (range, 0 to 2) during active movement, and 1.4 (range, 0 to 3 ) during weight-bearing walking. Clinical and radiological outcomes are shown in Table 2.

\begin{tabular}{c|c|c|c|c|c|c}
\multicolumn{6}{c}{ Table 2. Clinical and Radiological Outcomes on Follow-up. } \\
\hline $\begin{array}{c}\text { Patient } \\
\text { No. }\end{array}$ & $\begin{array}{c}\text { Follow-up } \\
\text { (months) }\end{array}$ & $\begin{array}{c}\text { OA- } \\
\text { Score }\end{array}$ & $\begin{array}{c}\text { AOFAS } \\
\text { Score }\end{array}$ & $\begin{array}{c}\text { VAS } \\
\text { Score } \\
\text { (rest) }\end{array}$ & $\begin{array}{c}\text { VAS Score } \\
\text { (active } \\
\text { movement) }\end{array}$ & $\begin{array}{c}\text { VAS Score } \\
\text { (weight-bearing } \\
\text { walking) }\end{array}$ \\
\hline 1 & 30 & 0 & 86 & 0 & 0 & 0 \\
\hline 2 & 24 & 1 & 89 & 1 & 1 & 2 \\
\hline 3 & 35 & 0 & 98 & 0 & 0 & 0 \\
\hline 4 & 32 & 0 & 92 & 0 & 0 & 0 \\
\hline 5 & 36 & 1 & 82 & 1 & 2 & 3 \\
\hline 6 & 27 & 0 & 91 & 0 & 0 & 0 \\
\hline 7 & 48 & 1 & 84 & 1 & 2 & 3 \\
\hline 8 & 40 & 2 & 82 & 2 & 2 & 3 \\
\hline 9 & 38 & 1 & 85 & 1 & 1 & 2 \\
\hline 10 & 52 & 0 & 89 & 0 & 0 & 1 \\
\hline
\end{tabular}

Abbreviations:OA, osteoarthritis; AOFAS, American orthopaedic foot and ankle society; VAS, visual analogue scale.

\section{DISCUSSION}

Pilon fractures are caused by high-energy or, more rarely, low-energy trauma. ${ }^{17,18}$ These fractures result from a combination of axial compressive forces and torsional forces. In high energy fracture patterns axial forces predominate, while torsional forces account for low energy pilon fractures. The fracture patterns Acta Ortop Bras. 2014;22(1):48-53 depend on the weight of the two different causative factors of injury. On the basis of CT scans, Topliss et al. ${ }^{11}$ classified pilon fractures into sagittal and coronal families. The former followed a high energy injury in young patients, while the latter followed a low energy trauma in old patients. In their cases, $56 \%$ of all pilon fractures belonged to the coronal family, in which the posterior split-type fractures (posterior pilon fractures) accounted for $10 \%$. Calori et al. ${ }^{19}$ stated that pilon fractures could be partial or complete, the partial could be divided into anterior and posterior, and the posterior were usually only one large fragment. Mast et al. ${ }^{20}$ thought that axial load combined with rotational load could cause a large posterior plafond fracture and viewed these injuries as pilon fractures because of the involvement of a large weight-bearing surface. Huber et al..$^{5}$ introduced the term "trimalleolar pilon fractures" and stated that the bigger the posterior malleolar fragment and the further the medial extension of the fracture line, the closer seems the relationship to pilon fractures. On the basis of these studies, fractures of the posterior pilon are not uncommon. But a definitive and direct description of this kind of fractures can be found in only a few articles. Hansen ${ }^{6}$ used the term "posterior pilon" to describe severe trimalleolar fractures involving the posterior tibial plafond and containing an impacted fragment. Amorosa et al. ${ }^{3}$ concluded that a posterior pilon fracture, having a longer postoperative course and slower functional recovery than standard ankle fractures, was a unique fracture pattern. It involved a large posterior malleolar fragment along with associated medial and lateral malleolar fractures, and marginal impaction and comminution of the posterior fragments could be found by CT scans or intraoperative findings. We agree with Amorosa's assessment and the injury pattern of our patients in this cohort was consistent with their description. Moreover, proximal displacement of the posterior malleolar fragments and subluxation of the talus were found in all our cases.

Other authors have described this fracture pattern, but they reported this kind of fractures as ankle fractures. Weber ${ }^{9}$ reported on 10 patients with trimalleolar fractures with multifragmentary posteromedial fractures of the distal tibia. Nine of 10 patients in their cases had axial impaction of osteochondral fragments. Gardner et al..$^{21}$ thought this kind of fracture could not be classified in the Lauge-Hansen system and was an ankle fracture variant. Wang et al..$^{10}$ reported on 12 patients with trimalleolar fractures with involvement of the entire posterior plafond. In their cohort, osteochondral impaction occurred in 3 patients. The fracture patterns these authors described are similar to that seen in our cases and are in accordance with the characteristics of a posterior pilon. Although Abdelgawad et al. ${ }^{22}$ thought that the posterior pilon described by Amorosa et al. ${ }^{3}$ would still be referred to as a trimalleolar fracture by most orthopaedic surgeons, we support the opinion of Amorosa et al. ${ }^{3}$ and insist on the existence of the posterior pilon fractures. In our opinion, the impacted fragments can only be caused by the axial load, which is the main injury mechanism of pilon fractures. Besides, with the ankle in hyperplantarflexion, when the posterior tibial plafond is hit by the talus, there is enough space for the posterior fragment to displace proximally, thus not always leading to severe comminution of the fragment like in the classic pilon fractures. We hold that posterior pilon fractures belong to low-energy pilon fractures. 
Distinguishing posterior pilon fractures from posterior malleolar fractures is important because of the different therapeutic protocols and prognoses. Because plain radiographic films cannot provide enough information, CT scanning is necessary for identifying a posterior pilon fracture. A study of Büchler et al. ${ }^{23}$ showed that assessment of the fracture anatomy at the posterior tibial margin based on plain radiography underestimated by far the posteromedial extension of the fracture line, the impaction of the posterior fracture edge, and additional impacted osteochondral fragments. In our cohort, preoperative CT evaluation was available in all patients. Posterior marginal impaction or impacted fragments were found in all of our patients. In addition, the CT scan also helped to identify the predominant location of the fragments. If the fragment was posterolateral, then only one incision was used for both tibial and fibular fixation. If there were multiple fragments both posterolaterally and posteromedially, an additional posteromedial approach was used.

Many techniques have been reported for reduction of the impacted osteochondral fragments of the posterior tibial plafond. Through the posteromedial approach, Bios et al. ${ }^{4}$ reflected the medial malleolus distally to expose the tibiotalar joint and allow direct reduction of the articular surface. But Weber ${ }^{9}$ thought the turnover of the posteromedial fragment through a posteromedial approach would pull the talus into posterior dislocation making it impossible to reduce the impacted osteochondral fragments. They advocated reducing the impacted fragments through a posterolateral approach. In accordance with their technique, we reduced the osteochondral fragments through the posterolateral approach. The impacted fragments were fixed by being squeezed underneath the reduced posterior fragment. Here we used a $1.5 \mathrm{~mm} \mathrm{~K}$-wire to fix the impacted fragment temporarily. Care should be taken to avoid damage of the anterior neurovascular bundles.

Anatomical reduction of the posterior malleolus is the goal of surgical treatment of posterior pilon fractures to limit the articular degenerative changes and improve the outcomes. The posterolateral approach could provide excellent exposure and direct visualization of the large posterior articular fragment as well as the smaller impacted fragments, thus allowing better reduction. A comparative study showed that the direct reduction through the posterolateral approach produced an anatomical reduction in 25 of 30 cases (83\%), while the indirect reduction and subsequent fixation through the anterior approach resulted in an anatomical reduction in 8 of 30 cases $(27 \%) .{ }^{5}$ Moreover, the lateral malleolar fracture can be reduced and fixed through the same incision. In our cases, we chose the posterolateral approach to reduce the fractures. Meanwhile, in some cases, an additional posteromedial approach was used to fix the posteromedial fragment. Under direct visualization, sound reduction was achieved in all of our cases. Moreover, an immediate postoperative CT scanning was done to assess the quality of the reduction. The postoperative CT scans showed that the stepoff of the articular surface was limited to $1 \mathrm{~mm}$. At two-year follow-up, the mean OA-score was 0.6, which was comparable with other reports. 2,10 Most of patients showed congruent ankle joints with no obvious degenerative changes.

With regard to the fixation of the posterior fragments, the choices include anteroposterior screw-fixation, posteroanterior screw-fixation and posterior buttress plating. A recent survey showed that trauma-trained surgeons were significantly more likely to choose buttress plating compared to screw-only fixation. ${ }^{24}$ Mingo-Robinet et al. ${ }^{1}$ reported 6 of 15 fractures (40\%), with the posterior malleolar fragment fixated by anterior to posterior screws, had failed fixations. Other authors ${ }^{25-27}$ stated that posteroanterior screw-fixation provides biomechanically superior fixation than does anteroposterior screw-fixation. In the study of Huber et al., ${ }^{5}$ buttress plating produced good stability, while one patient with anteroposterior screw-fixation had secondary displacement leading to two reoperations and a poor result. Other reports also support the application of a buttress plate for fixation of the posterior malleolar fragment because of the stability of such fixation and good long-term outcomes., ${ }^{4,22}$ However, the superiority of buttress plating versus screw fixation still needs biomechanical proofs. In addition, most of these reports were about the posterior malleolar fractures. Regarding the posterior pilon fractures, Amorosa et al. ${ }^{3}$ used posterior to anterior screws to fix the posterior malleolar fragments and attained good stabilization. However, postoperative splint immobilization was employed in their cases. In our cohort, we chose buttress plating and all fractures gained stable fixation. Moreover, no external splints were used and active range of motion exercises were started 24 hours postoperatively. No loss of reduction or fixation failure occurred. In our opinion, because the injury mechanism of a posterior pilon fracture contains the component of axial forces and shearing forces, leading to large displaced posterior malleolar fragments and impacted fragments, application of buttress plating is deemed necessary. Besides, benefitting from the stable fixation, buttress plating allows earlier motion of the ankle joint, thus helping recovery of the articular cartilage ${ }^{28}$ and avoiding the occurrence of articular stiffness following plaster immobilization. At two-year follow-up, the functional results were favorable with a mean AOFAS score of 87.8. The VAS scores were low. Severe ankle pain and swelling or articular stiffness was not found in our cases. All patients were satisfied and returned to their normal work and leisure activities.

Limitations of this study include the intrinsic weakness of a retrospective study, a small cohort, and lack of powerful statistical data to reveal the advantage of buttress plating. In addition, although we chose the AOFAS score, the OA-score and the VAS score to assess the outcomes, we could not thoroughly compare the results with other reports. This could be partly attributed to the facts that most of the posterior pilon fractures were mixed up with posterior malleolar fractures to be reported. Although some other limitations might exist in this study, we believe that our patients gained proper management and the clinical outcomes were favorable.

\section{CONCLUSIONS}

The posterior pilon fractures are low-energy pilon fractures. CT scanning is helpful for evaluating a posterior pilon fracture. Anatomical reduction and stable fixation of a posterior pilon can be realized by direct manipulation through a posterolateral or combined approach. Buttress plating could provide stable fixation and allows earlier postoperative exercises without plaster immobilization, thus minimizing the risk of posttraumatic arthritis, although rigorous studies supporting this claim are pending. 


\section{REFERENCES}

1. Mingo-Robinet J, López-Durán L, Galeote JE, Martinez-Cervell C. Ankle fractures with posterior malleolar fragment: management and results. J Foot Ankle Surg. 2011;50(2):141-5.

2. De Vries JS, Wijgman AJ, Sierevelt IN, Schaap GR. Long-term results of ankle fractures with a posterior malleolar fragment. J Foot Ankle Surg. $2011 ; 44(3): 211-7$.

3. Amorosa LF, Brown GD, Greisberg J. A surgical approach to posterior pilon fractures. J Orthop Trauma. 2010;24(3):188-93.

4. Bois AJ, Dust W. Posterior fracture dislocation of the ankle: technique and clinical experience using a posteromedial surgical approach. J Orthop Trauma. 2008;22(9):629-36.

5. Huber M, Stutz PM, Gerber C. Open reduction and internal fixation of the posterior malleolus with a posterior antiglide plate using a posterolateral approach- a preliminary report. Foot Ankle Surg. 1996;2(2):95-103.

6. Hansen ST. Functional Reconstruction of the foot and ankle. Philadelphia: Lippincott Williams \& Wilkins; 2000

7. Harper MC, Hardin G. Posterior malleolar fractures of the ankle associated with external rotation-abduction injuries. Results with and without internal fixation. J Bone Joint Surg Am. 1988;70(9):1348-56.

8. Karachalios T, Roidis N, Karoutis D, Bargiotas K, Karachalios GG. Trimalleolar fracture with a double fragment of the posterior malleolus: a case report and modified operative approach to internal fixation. Foot Ankle Int. 2001;22(2):144-9.

9. Weber M. Trimalleolar fractures with impaction of the posteromedial tibial plafond: implications for talar stability. Foot Ankle Int. 2004;25(10):716-27.

10. Wang L, Shi ZM, Zhang CQ, Zeng BF. Trimalleolar fracture with involvement of the entire posterior plafond. Foot Ankle Int. 2011;32(8):774-81.

11. Topliss CJ, Jackson M, Atkins RM. Anatomy of pilon fractures of the distal tibia. J Bone Joint Surg Br. 2005;87(5):692-7.

12. Oestern HJ, Tscherne H. Pathophysiology and classification of soft tissue damage in fractures. Orthopade. 1983;12(1):2-8.

13. Kitaoka HB, Alexander IJ, Adelaar RS, Nunley JA, Myerson MS, Sanders M. Clinical rating systems for the ankle-hindfoot, midfoot, hallux, and lesser toes. Foot Ankle Int. 1994;15(7):349-53.
14. Xu HL, Li X, Zhang DY, Fu ZG, Wang TB, Zhang PX, et al. A retrospective study of posterior malleolus fractures. Int Orthop. 2012;36(9):1929-36.

15. Katz J, Melzack R. Measurement of pain. Surg Clin North Am. 1999;79(2):231-52.

16. Langenhuijsen JF, Heetveld MJ, Ultee JM, Steller EP, Butzelaar RM. Results of ankle fractures with involvement of the posterior tibial margin. J Trauma 2002;53(1):55-60.

17. Borrelli J Jr, Ellis E. Pilon fractures: assessment and treatment. Orthop Clin North Am. 2002;33(1):231-45

18. Crist BD, Khazzam M, Murtha YM, Della Rocca GJ. Pilon fractures: advances in surgical management. J Am Acad Orthop Surg. 2011;19(10):612-22.

19. Calori GM, Tagliabue L, Mazza E, de Bellis U, Pierannunzii L, Marelli BM, et al. Tibial pilon fractures: which method of treatment? Injury. 2010;41(11):1183-90.

20. Mast JW, Spiegel PG, Pappas JN. Fractures of the tibial pilon. Clin Orthop Relat Res. 1988;(230):68-82.

21. Gardner MJ, Boraiah S, Hentel KD, Helfet DL, Lorich DG. The hyperplantarflexion ankle fracture variant. J Foot Ankle Surg. 2007;46(4):256-60.

22. Abdelgawad AA, Kadous A, Kanlic E. Posterolateral approach for treatment of posterior malleolus fracture of the ankle. J Foot Ankle Surg. 2011;50(5):607-11.

23. Büchler L, Tannast M, Bonel HM, Weber M. Reliability of radiologic assessment of the fracture anatomy at the posterior tibial plafond in malleolar fractures. J Orthop Trauma. 2009;23(3):208-12.

24. Gardner MJ, Streubel PN, McCormick JJ, Klein SE, Johnson JE, Ricci WM. Surgeon practices regarding operative treatment of posterior malleolus fractures. Foot Ankle Int. 2011;32(4):385-93.

25. Tornetta P 3rd, Ostrum RF, Trafton PG. Trimalleolar ankle fracture. J Orthop Trauma. 2001;15(3):588-90

26. Talbot M, Steenblock TR, Cole PA. Posterolateral approach for open reduction and internal fixation of trimalleolar ankle fractures. Can J Surg. 2005;48(6):487-90.

27. Heim UF. Trimalleolar fractures: late results after fixation of the posterior fragment. Orthopedics. 1989;12(8):1053-9.

28. Salter RB, Simmonds DF, Malcolm BW, Rumble EJ, MacMichael D, Clements ND. The biological effect of continuous passive motion on the healing of full-thickness defects in articular cartilage. An experimental investigation in the rabbit. J Bone Joint Surg Am. 1980;62(8):1232-51. 